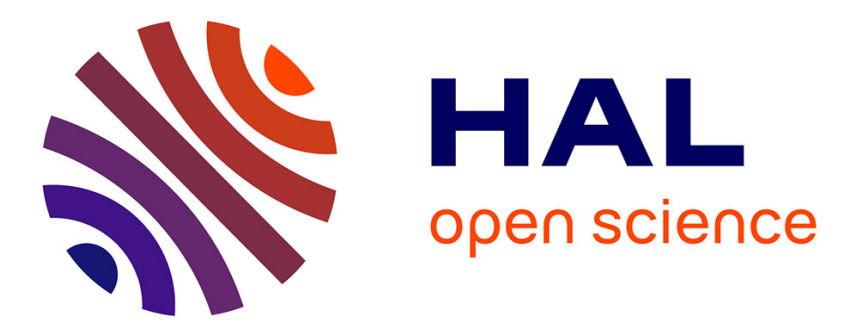

\title{
Hunt's colorimetric effect from a quantum measurement viewpoint
}

Michel Berthier, Edoardo Provenzi

\section{To cite this version:}

Michel Berthier, Edoardo Provenzi. Hunt's colorimetric effect from a quantum measurement viewpoint. Geometric Science of Information, Jul 2021, Paris, France. hal-03276158

\section{HAL Id: hal-03276158 \\ https://hal.science/hal-03276158}

Submitted on 1 Jul 2021

HAL is a multi-disciplinary open access archive for the deposit and dissemination of scientific research documents, whether they are published or not. The documents may come from teaching and research institutions in France or abroad, or from public or private research centers.
L'archive ouverte pluridisciplinaire HAL, est destinée au dépôt et à la diffusion de documents scientifiques de niveau recherche, publiés ou non, émanant des établissements d'enseignement et de recherche français ou étrangers, des laboratoires publics ou privés. 


\title{
Hunt's colorimetric effect from a quantum measurement viewpoint
}

\author{
Michel Berthier*1 and Edoardo Provenzi ${ }^{\dagger 2}$ \\ ${ }^{1}$ Laboratoire MIA, Université de La Rochelle, Avenue Albert Einstein, 17031 La \\ Rochelle BP 33060, France, Tel.: +33(0)5 46458605 \\ ${ }^{2}$ Université de Bordeaux, CNRS, Bordeaux INP, IMB, UMR 5251, F-33400, 351 \\ Cours de la Libération, Talence, France, Tel.: +33(0)5 40006437
}

\begin{abstract}
The main aim of this paper is to describe how the colorimetric phenomenon known as 'Hunt effect' can be understood in the quantum framework developed by the authors to model color perception of trichromatic observers. In classical colorimetry, the most common definition of the Hunt effect is that the colorfulness of a color increases with its luminance, however several other definitions are available. The need to establish a unique and precise characterization of the features involved in the Hunt effect led us to propose novel mathematical definitions of colorimetric attributes. Within the newly established nomenclature, we show how the Hunt effect can be rigorously explained thanks to the duality between quantum states and effects.
\end{abstract}

\section{Introduction}

The main aim of this paper is to explain the well known Hunt colorimetric effect in the quantum framework first introduced in [4] and then refined and further formalized in [2] and [3]. Due to space limitation, here we will recall only the elements of this framework that are strictly necessary for the following.

The work of the founding fathers of colorimetry, Newton, Maxwell, Grassmann and von Helmholtz, was elegantly resumed by Schrödinger in 13 in a set of axioms which imply that the space of perceptual colors $\mathcal{C}$ is a 3 -dimensional convex regular cone. In [12, Resnikoff added an homogeneity axiom which implied that $\mathcal{C}$, as a set, can only take two forms: either $\mathcal{C}_{1}=\mathbb{R}^{+} \times \mathbb{R}^{+} \times \mathbb{R}^{+}$ or $\mathcal{C}_{1}=\mathbb{R}^{+} \times \mathbf{H}$, where $\mathbf{H}$ is a 2-dimensional hyperbolic space, see also [11.

The core of our approach is a mathematical axiom called trichromacy axiom that summarizes the set of Schrödinger's and Resnokoff's axioms into a single one: the space of perceptual colors is the positive cone $\mathcal{C}$ of a formally real Jordan algebra of real dimension 3. Jordan algebras are commutative but non necessarily associative algebras used as the foundation of the algebraic formulation of quantum theory developed by Jordan, von Neumann and Wigner.

Using the classification theorem of finite dimensional formally real Jordan algebras one can check that $\mathcal{C}_{1}$ is the positive cone of the commutative and associative Jordan algebra $\mathbb{R} \oplus \mathbb{R} \oplus \mathbb{R}$, and $\mathcal{C}_{2}$ is $\mathcal{H}^{+}(2, \mathbb{R})$, the cone of positive-definite $2 \times 2$ real matrices, which is the positive cone of the commutative but non-associative Jordan algebra $\mathcal{H}(2, \mathbb{R})$ of $2 \times 2$ real symmetric matrices equipped with the Jordan matrix product. The first case corresponds to the classical CIE (Commission International de l'Éclairage) colorimetry, whereas the second case corresponds to a non classical colorimetric theory. From now on, we focus only on the latter case and we will give several motivations to consider it as a genuine quantum theory of color perception.

\footnotetext{
*michel.berthier@univ-lr.fr

†edoardo.provenzi@math.u-bordeaux.fr
} 
Among other reasons, a striking fact leads us to think that the classical CIE approach should be abandoned: all CIE color spaces are founded on either the RGB or the XYZ spaces, which are built with the only aim at codifying the output of the three retinal cone photoreceptors through values that are obtained via color matching experiments. The flaw in this procedure is that the outcome of those experiments is the result of the action of the whole human visual system, not only of the cones. Moreover, the fundamental processing known as Hering's opponency, due to the action of ganglion cells, which detect and magnify the differences between the outputs of the different cones, is not incorporated in the RGB or XYZ spaces. This fact is confirmed by our interpretation of the classical color space as the positive cone of the commutative and associative Jordan algebra $\mathbb{R} \oplus \mathbb{R} \oplus \mathbb{R}$. Instead, as we are going to recall, if we consider as color space the positive cone of the commutative but non-associative Jordan algebra $\mathcal{H}(2, \mathbb{R})$ and we use the self-duality of this cone, then we can intrinsically account for both the trichromatic and Hering's opponency stages.

The key to exhibit Hering's opponency is to exploit the Jordan algebras isomorphism between $\mathcal{H}(2, \mathbb{R})$ and the spin factor $\mathbb{R} \oplus \mathbb{R}^{2}$, which implies that the trichromacy cone $\mathcal{C}$ can be identified with the 3-dimensional future lightcone:

$$
\mathcal{L}^{+}=\left\{(\alpha+\mathbf{v}) \in \mathbb{R} \oplus \mathbb{R}^{2}: \alpha>0,\|(\alpha+\mathbf{v})\|_{\mathcal{M}}>0\right\},
$$

where $\|(\alpha+\mathbf{v})\|_{\mathcal{M}}^{2}=\alpha^{2}-\|\mathbf{v}\|^{2}$ denotes the squared Minkowski norm, $\|$ \| being the Euclidean norm. The state space $\mathcal{S}$ of a rebit, the real analog of the usual (complex) qubit, can be identified with $\left\{\mathbf{v} \in \mathbb{R}^{2}:\|\mathbf{v}\| \leq 1\right\}$ and, crucially, see e.g. [4, 2, 3], it can be naturally embedded in the closure $\overline{\mathcal{L}^{+}}$of $\mathcal{L}^{+}$via the map

$$
\mathcal{S}=\left\{\mathbf{v} \in \mathbb{R}^{2}:\|\mathbf{v}\| \leq 1\right\} \ni \mathbf{v} \mapsto \frac{1}{2}(1+\mathbf{v}) \in \mathcal{D}_{1 / 2}=\left\{(\alpha+\mathbf{v}) \in \overline{\mathcal{L}^{+}}: \alpha=1 / 2\right\} .
$$

The properties of this so-called Hering's rebit allow us to give a meaningful mathematical interpretation of Hering's color opponency. Let us consider the embedding

$$
\mathcal{S} \ni \mathbf{v}=(r \cos \theta, r \sin \theta) \mapsto \rho(\mathbf{v})=\rho(r, \theta)=\frac{1}{2}\left(\begin{array}{cc}
1+r \cos \theta & r \sin \theta \\
r \sin \theta & 1-r \cos \theta
\end{array}\right) \in \mathcal{D} \mathcal{M},
$$

where, $0 \leq \theta<2 \pi$ and $\mathcal{D} \mathcal{M}$ denotes the space of density matrices of $\mathcal{H}(2, \mathbb{R})$. One can easily checks that

$$
\rho(r, \theta)=\rho_{0}+\frac{r \cos \theta}{2}[\rho(1,0)-\rho(1, \pi)]+\frac{r \sin \theta}{2}[\rho(1, \pi / 2)-\rho(1,3 \pi / 2)] .
$$

From a colorimetric viewpoint, this decomposition means that the generic quantum chromatic state represented by a density matrix $\rho(r, \theta)$ can be seen as the superposition of the maximal von Neumann entropy state $\rho_{0}=\rho(0,0)$ (see section 3 for more information), which represents the achromatic state, with two diametrical oppositions of pure hues (or pure tints). We refer to [3] or 2. for further information, especially for what concerns the hyperbolic metric aspects of this theory.

The fact that a rebit system emerges naturally from the sole trichromacy axiom is one of the reasons that led us to consider this non-classical colorimetric theory as a quantum-like theory in which we assume that:

- a visual scene is a setting where we can perform psycho-visual measurements in conditions that are as isolated as possible from external influences;

- a perceptual chromatic state is represented by the preparation of a visual scene for psychovisual experiments;

- a perceptual color is the observable identified with a psycho-visual measurement performed on a given perceptual chromatic state.

Of course, the instrument used to measure the observables is the visual system of a human and not a technological device used to perform physical experiments.

So far we have recalled basic information from our quantum-like theory of color perception, in the next sections we are going to introduce in this framework the concept of quantum effect which will allow us to describe the Hunt effect from a mathematical point of view. 


\section{States and effects in colorimetry}

Operationally speaking, the concept of a state in quantum mechanics refers to an ensemble of identically prepared systems, while the concept of effect refers to a measurement apparatus that produces an outcome. The duality between states and effects means essentially that when a state and an effect are specified, one can compute a probability distribution which is the only meaningful information that we can obtain about the experiment. In other words, quantum mechanics deals with a collection of possible preparations and measurements, which can be combined together to form an experiment leading to a probability distribution of measurement outcomes 7 .

In order to translate in mathematical terms what just stated, let us introduce the state cone:

$$
\mathcal{C}(\mathcal{S})=\{\alpha(1, \mathbf{v}), \alpha \geq 0, \mathbf{v} \in \mathcal{S}\}
$$

that, using the embeddings (2) and (3), can be easily checked to satisfy

$$
\mathcal{C}(\mathcal{S}) \simeq\left\{2 \alpha\left[\frac{1}{2}(1+\mathbf{v})\right], \alpha \geq 0, \mathbf{v} \in \mathcal{S}\right\}=\overline{\mathcal{L}^{+}}
$$

and

$$
\mathcal{C}(\mathcal{S}) \simeq\{2 \alpha \rho(\mathbf{v}), \alpha \geq 0, \mathbf{v} \in \mathcal{S}\}=\overline{\mathcal{H}^{+}(2, \mathbb{R})},
$$

where $\overline{\mathcal{H}^{+}(2, \mathbb{R})}$ is the set of positive semi-definite $2 \times 2$ real matrices.

Consequently, since the positive cone of a formally real Jordan algebra is self dua 1 the state cone $\mathcal{C}(\mathcal{S})$ is itself self dual, i.e. $\mathcal{C}^{*}(\mathcal{S})=\mathcal{C}(\mathcal{S})$. The effect space of $\mathcal{S}$ is defined as follows:

$$
\mathcal{E}(\mathcal{S})=\left\{e \in \mathcal{C}^{*}(\mathcal{S}), e \leq I d\right\},
$$

where $I d$ is the unit element of either $\mathbb{R} \oplus \mathbb{R}^{2}$ or $\mathcal{H}(2, \mathbb{R})$, accordingly to the identification (6) or (7), respectively. To obtain a geometric description of $\mathcal{E}(\mathcal{S})$, one can consider an effect $e=\alpha(1, \mathbf{v})$ as an element $2 \alpha \rho(\mathbf{v})$ of $\overline{\mathcal{H}^{+}(2, \mathbb{R})}$ that satisfies the two conditions $\operatorname{Tr}(I d-e) \geq 0$ and $\operatorname{det}(I d-e) \geq 0$. Direct computations show that

$$
\mathcal{E}(\mathcal{S})=\left\{\alpha\left(1, v_{1}, v_{2}\right), \alpha \in[0,1], v_{1}^{2}+v_{2}^{2} \leq \min \left\{\frac{(1-\alpha)^{2}}{\alpha^{2}}, 1\right\}\right\} .
$$

By using self duality, a straightforward computation leads to

$$
\mathcal{E}(\mathcal{S}) \simeq\left\{e=\left(\begin{array}{l}
a_{0} \\
a_{1} \\
a_{2}
\end{array}\right): e\left(1, v_{1}, v_{2}\right)=a_{0}+a_{1} v_{1}+a_{2} v_{2} \in[0,1], \forall\left(v_{1}, v_{2}\right) \in \mathcal{S}\right\} .
$$

Every effect is thus uniquely associated to a $[0,1]$-valued affine function (denoted again with e for simplicity) on the set of states. This is in fact the simplest way to associate a probability to each state. Eq. 9 implies that the effect space $\mathcal{E}(\mathcal{S})$ can be represented as a double cone in the 3-dimensional Minkowski space, as shown in Fig. 1.

Remarkably, this double cone coincides with that introduced in [5] as a representation of a so-called perceptual color space whose shape results from the description of the physiological mechanisms of neural coding of colors. Since this perceptual color space is, up to our knowledge, the only color space that has been proposed to take into account the spectrally opponent and non-opponent interactions of Hering's opponency, we consider this as particularly relevant.

All the previous considerations lead us quite naturally to introduce the following definition: $a$ color effect (also called a perceived color in [2]), is an element $c$ of the effect space $\mathcal{E}(\mathcal{S})$.

\footnotetext{
${ }^{1}$ Given a formally real Jordan algebra $\mathcal{A}$, its positive cone agrees with its the dual cone $\mathcal{C}^{*}:=\{a \in \mathcal{A}: \forall b \in$ $\mathcal{C},\langle a, b\rangle>0\}$, where $\langle a, b\rangle=\operatorname{Tr}\left(L_{a \circ b}\right), L_{a \circ b}(c)=(a \circ b) \circ c, \forall c \in \mathcal{A}$. $\mathcal{C}^{*}$ can be identified with the set of positive linear functionals on $\mathcal{A}$.
} 


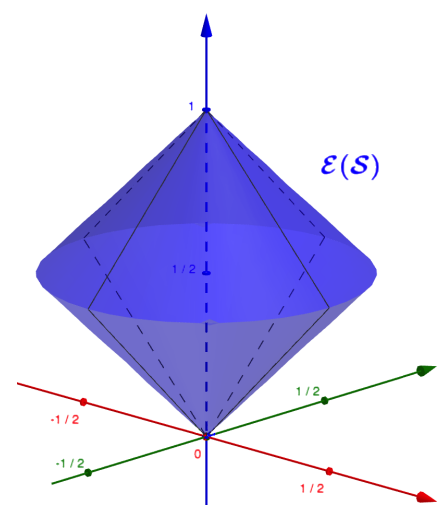

Figure 1: The convex double cone representing $\mathcal{E}(\mathcal{S})$.

\section{Colorimetric attributes of color effects}

In order to give a meaningful mathematical formulation of Hunt's effect it is necessary to introduce supplementary definitions related to the colorimetric attributes of a color effect. These definitions involve the notion of entropy which aims at measuring the mathematical expectation of the possible information gain about a given system. Dealing with quantum-like states, one usually considers the von Neumann entropy $S(\rho)$ of a density matrix $\rho$, which in our context, see e.g. [1], can be taken to be:

$$
S(\rho)=-\operatorname{Tr}(\rho \circ \log \rho),
$$

where $\circ$ denotes the Jordan product of $\mathcal{H}(2, \mathbb{R})$. By using the parametrization $\rho(r, \theta)$ it can be checked that the von Neumann entropy is given by:

$$
S(\rho)=-\log \left(\frac{1-r}{2}\right)^{\frac{1-r}{2}}\left(\frac{1+r}{2}\right)^{\frac{1+r}{2}},
$$

with $S(1)=\lim _{r \rightarrow 1^{-}} S(r)=0$. Pure state density matrices are characterized by a null entropy: pure states provide the maximum of information. The density matrix $\rho_{0}=\rho(0,0)$ is the unique density matrix with maximal von Neumann entropy: the state $(0,0) \in \mathcal{S}$ is the state of maximal entropy that provides no chromatic information.

Let $\mathbf{v}=\left(v_{1}, v_{2}\right)=(r \cos \theta, r \sin \theta)$ be a state of $\mathcal{S}$. Given the color effect $c=\alpha\left(1, v_{1}, v_{2}\right) \simeq$ $2 \alpha \rho(r, \theta)$, we call the real $\alpha, 0 \leq \alpha \leq 1$, the magnitude of $c$. This magnitude is nothing but the result of the evaluation of $c$ on the maximal entropy state $\rho_{0}$ :

$$
\alpha=\langle c\rangle_{\rho_{0}}=\operatorname{Tr}\left(\rho_{0} \circ 2 \alpha \rho(r, \theta)\right) .
$$

The extreme simplicity of this definition stands out even more if compared to the plethora of definitions of chromatic attributes corresponding to $\alpha$ in classical colorimetry. Readers can refer for instance to the paper [9] to make up their own mind about this topic.

If we divide the effect $c$ by $2 \alpha$, we obtain the density matrix $\rho(r, \theta)$ that characterizes the chromatic state of $c$. As its name suggests, this chromatic state retains only the chromatic information of $c$. In order to recover this latter, one can perform measurements using the two Pauli-like matrices of Hering's rebit:

$$
\sigma_{1}=\left(\begin{array}{cc}
1 & 0 \\
0 & -1
\end{array}\right), \sigma_{2}=\left(\begin{array}{ll}
0 & 1 \\
1 & 0
\end{array}\right)
$$

In fact, the expected values

$$
\left\langle\sigma_{1}\right\rangle_{\rho(r, \theta)}=\operatorname{Tr}\left(\rho(r, \theta) \circ \sigma_{1}\right)=r \cos \theta, \quad\left\langle\sigma_{2}\right\rangle_{\rho(r, \theta)}=\operatorname{Tr}\left(\rho(r, \theta) \circ \sigma_{2}\right)=r \sin \theta,
$$

give the degree of opposition between the two pairs of pure chromatic states involved in Hering's opposition, i.e. the two spectrally opponent interactions of the neural coding of colors. Let us 
insist on the fact that, in practice, performing such measurements is not straightforward: the matrices $\sigma_{1}$ and $\sigma_{2}$ refer to mechanisms implemented by the sensory system of a human being and not by technological devices. The reader can refer for instance to 8 for related psycho-visual experiment descriptions. Due to the lack of space, we do not investigate here the fascinating problem that consists of explaining the relation between the well-known phenomenon of color indistinguishability, illustrated by MacAdam ellipses, and the measurement uncertainty inherent to the fact that the matrices $\sigma_{1}$ and $\sigma_{2}$ do not commute. This will be the subject of forthcoming studies.

Since the von Neumann entropy $S(\rho)$ of $\rho=\rho(r, \theta)$ allows us to measure the purity degree of the chromatic state $\rho$ of the color effect $c$, it seems natural to relate it with the classical notion of saturation widely used in colorimetry by defining the saturation of the color effect $c$ as follows

$$
\sigma(c)=\frac{\log (2)-S(\rho)}{\log (2)},
$$

the graph of $\sigma(c)$ is depicted in Fig. 2.

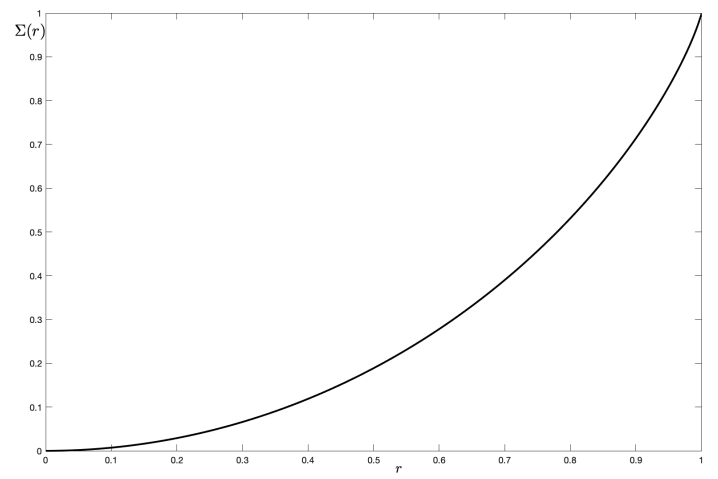

Figure 2: A proposal for the saturation of a color effect from built from the von Neumann entropy of its chromatic state.

Notice that this proposal is the simplest one which agrees with the classical constraints: 0 for achromatic colors and 1 for pure (spectral) colors.

The final colorimetric attribute that remains to be defined is hue. Actually, this is the only definition that remains identical to that of classical colorimetry, as we will consider the angle $\theta$ as the hue of the color effect $c$.

\section{Hunt's colorimetric effect}

In order to introduce this effect (which is not a quantum effect, but a psycho-visual effect!) we quote the classical reference on colorimetry [6]: 'The color appearances of objects change significantly when the overall luminance level changes. The Hunt effect can be summarized by the statement that colorfulness of a given stimulus increases with luminance level'.

To give an idea about the extreme difficulty of discussing color in words, let us quote how the terms involved in this description of Hunt's effect are defined in [6]: 'Colorfulness is the attribute of a visual perception according to which the perceived colour of an area appears to be more or less chromatic. Chroma is the colorfulness of an area judged as a proportion of the brightness of a similarly illuminated area that appears white or highly transmitting. Saturation is the colorfulness of an area judged in proportion to its brightness'.

In spite of the involved way of dealing with these concepts, it is clear that the Hunt effect reveals that the colorimetric attributes are not independent. This has the immediate consequence that color spaces such as XYZ, RGB, HSV, HCV and so on (see e.g. [6]), in which color is encoded 
by three uncorrelated values, are not suitable to represent perceived colors. Instead, the category of color spaces as CIELab, CIECAM and so on, issued from the so-called color appearance models, are built in such a way that color coordinates are correlated and, in fact, experimental data about the Hunt effect are available, see [10. Nonetheless, also these spaces come from XYZ and so their account of Hunt's effect is a posteriori and with several ad-hoc parameters difficult to control.

Contrary to this, the quantum-like approach that we have described above allows to derive the Hunt effect intrinsically and rigorously. To show this, consider a visual scene and a chromatic state with density matrix $\rho=\rho(r, \theta)$, i.e. a preparation of the visual scene for psycho-visual experiments. Let $c=2 \alpha \rho(r, \theta)$ be the corresponding color effect, i.e. the observable identified with a psycho-visual measurement performed on $\rho$. Suppose that $c$ is adapted to the maximal entropy state $\rho_{0}$, then, by direct computation, we have that the evaluation of $c$ on $\rho_{0}$ and on the chromatic state $\rho$ are, respectively, the magnitude $\alpha$ and

$$
\langle c\rangle_{\rho}=\operatorname{Tr}(\rho \circ c)=\alpha\left(1+r^{2}\right) .
$$

Let us discuss particular cases in order to understand this evaluation.

- If $\alpha=1 / 2$ then $\langle c\rangle_{\rho}=\left(1+r^{2}\right) / 2$. This evaluation holds for every $\rho$ and $\langle c\rangle_{\rho}$ is maximal, and equal to 1 , when $\rho$ is a pure chromatic state, i.e. when $r=1$.

- If $\alpha<1 / 2$ then $\langle c\rangle_{\rho}=\alpha\left(1+r^{2}\right) / 2$ holds for every $\rho$ and $\langle c\rangle_{\rho}<1$ for every $\rho$. It is maximal, and equal to $2 \alpha<1$, when $r=1$, i.e. for pure chromatic states.

- If $\alpha>1 / 2$, then the evaluation $\langle c\rangle_{\rho}$ can only be performed when $\rho$ is such that $r^{2} \leq(1-\alpha) / \alpha$. It is maximal, and equal to 1 , when $r^{2}=(1-\alpha) / \alpha$. For instance, if $\alpha=2 / 3$, then $\langle c\rangle_{\rho}=1$ when $r=\sqrt{2} / 2$.

Now, the chromatic state $\rho_{1}=\rho(\sqrt{2} / 2, \theta)$ is perceived by the effect $c_{1}=4 / 3 \rho_{1}$ in the same way as the chromatic state $\rho_{2}=\rho(1, \theta)$ is perceived by the effect $c_{2}=\rho_{2}$ since $\left\langle c_{1}\right\rangle_{\rho_{1}}=\left\langle c_{2}\right\rangle_{\rho_{2}}=1$. This means that the non-pure chromatic state $\rho_{1}$ is perceived in the same way as the a pure chromatic state $\rho_{2}$, and thus, it is perceived more saturated than it really is. Consequently, increasing the magnitude $\alpha$ from $1 / 2$ to $2 / 3$ increases the perceived saturation of the chromatic state $\rho_{1}$. Note that when $\alpha=1$, the color effect $c$ is the so-called unit effect that gives the same response equal to 1 on all states, this is the mathematical translation of the glare limit.

\section{Discussion and future perspectives}

We have shown mathematical objects known as effects in quantum theory allow us to mathematically account for the Hunt phenomenon of interdependence between (what are classically called) colorfulness and luminance without the need of introducing any exterior ad-hoc structures or parameters. What we find remarkable is that quantum concepts as rebit and effects, well-known in modern quantum information but less-known in ordinary quantum mechanics, seem to find a natural place in color perception theory.

\section{References}

[1] John C Baez. Division algebras and quantum theory. Foundations of Physics, 42(7):819-855, 2012.

[2] M. Berthier. Geometry of color perception. Part 2: perceived colors from real quantum states and Hering's rebit. Journal of Mathematical Neuroscience, 10(1):1-25, 2020.

[3] M. Berthier and E. Provenzi. From Riemannian trichromacy to quantum color opponency via hyperbolicity. Journal of Mathematical Imaging and Vision, DOI: 10.1007/s10851-02101023-5 (2021).

[4] M. Berthier and E. Provenzi. When geometry meets psycho-physics and quantum mechanics: Modern perspectives on the space of perceived colors. In GSI 2019, volume 11712 of Lecture Notes in Computer Science, pages 621-630. Springer, 2019. 
[5] Russell L De Valois and Karen D De Valois. Neural coding of color. 1997.

[6] M.D. Fairchild. Color appearance models. Wiley, 2013.

[7] Teiko Heinosaari and Mário Ziman. The mathematical language of quantum theory: from uncertainty to entanglement. Cambridge University Press, 2011.

[8] Dorothea Jameson and Leo M Hurvich. Some quantitative aspects of an opponent-colors theory. i. chromatic responses and spectral saturation. JOSA, 45(7):546-552, 1955.

[9] Frederick AA Kingdom. Lightness, brightness and transparency: A quarter century of new ideas, captivating demonstrations and unrelenting controversy. Vision research, 51(7):652673, 2011.

[10] M Ronnier Luo and Changjun Li. Cie color appearance models and associated color spaces. Colorimetry: Understanding the CIE system, pages 261-294, 2007.

[11] Edoardo Provenzi. Geometry of color perception. Part 1: Structures and metrics of a homogeneous color space. Journal of Mathematical Neuroscience, 10(1):1-19, 2020.

[12] H.L. Resnikoff. Differential geometry and color perception. Journal of Mathematical Biology, 1:97-131, 1974.

[13] E. Schrödinger. Grundlinien einer theorie der farbenmetrik im tagessehen (Outline of a theory of colour measurement for daylight vision). Annalen der Physik, 63(4):397-456; 481-520, 1920. 